08,04

\title{
Парамагнитные дефекты в кристаллах ZnSe, активированных ионами железа
}

\author{
() В.А. Важенин ${ }^{1}$, А.П. Потапов ${ }^{1}$, Г.С. Шакуров ${ }^{2}$, А.В. Фокин ${ }^{1}$, М.Ю. Артёмов ${ }^{1}$, \\ В.И. Козловский ${ }^{3,4}$, Ю.В. Коростелин ${ }^{3}$, Д.С. Пыталев \\ ${ }^{1}$ Уральский федеральный университет, \\ Екатеринбург, Россия \\ ${ }^{2}$ Казанский физико-технический институт им. Е.К. Завойского, \\ Казань, Россия \\ ${ }^{3}$ Физический институт им. П.Н. Лебедева РАН, \\ Москва, Россия \\ ${ }^{4}$ Национальный исследовательский ядерный университет „МИФИ“, \\ Москва, Россия \\ ${ }^{5}$ Институт спектроскопии РАН, \\ Москва, Россия \\ E-mail: Vladimir.Vazhenin@urfu.ru
}

(Поступила в Редакцию 6 апреля 2017 г.)

Исследованы спектры парамагнитного резонанса монокристаллов ZnSe, легированных железом. Кроме кубических центров $\mathrm{Fe}^{3+}$ и $\mathrm{Mn}^{2+}$, а также центров $\mathrm{Fe}^{2+}$ и $\mathrm{Cr}^{2+}$ обнаружены моноклинные комплексы $\mathrm{Fe}^{3+}$, локально компенсированные ионами $\mathrm{Cu}^{+}$. Также обнаружены и исследованы тригональные центры со спином 3/2. Измерены начальные расщепления моноклинных центров, определены параметры моноклинного и тригонального спиновых гамильтонианов. Обсуждается природа тригональных центров.

Работа выполнена в рамках государственного задания Минобрнауки России для Уральского федерального университета (3.6115.2017/8.9), Программы президиума РАН 1.26 в КФТИ КазНЦ РАН и при поддержке Программы повышения конкурентоспособности НИЯУ МИФИ. Измерения проведены на спектрометре трехсантиметрового диапазона Центра коллективного пользования „Современные нанотехнологии“ Уральского федерального университета и высокочастотном перестраиваемом спектрометре Казанского физико-технического института.

DOI: 10.21883/FTT.2017.10.44967.118

\section{1. Введение}

Кристаллы $\mathrm{ZnSe}$, активированные ионами группы железа, активно используются в качестве рабочей среды для широкополосных лазеров, излучающих в среднем ИК-диапазоне и работающих при комнатной температуре. Указанные приборы применяются в системах локации, для дистанционного зондирования, экологического контроля загрязнения окружающей среды, анализа газовых смесей, а также в различных медицинских целях [1]. О выборе для таких устройств кристаллов с оптимальными свойствами, обеспечивающими высокий квантовый выход люминесценции, подробно говорится в работе [1]. В ней также показаны возможности электронного парамагнитного резонанса (ЭПР) в определении зарядовых состояний легирующих ионов, вводимых в кристалл, в частности методом диффузии в условиях термодинамического равновесия фаз. Кроме того, ЭПР позволяет детектировать наличие в кристалле посторонних примесей. В случае кристаллов $\mathrm{ZnSe}: \mathrm{Fe}$ с точки зрения лазерных свойств очень важно соотношение концентраций ионов $\mathrm{Fe}^{+}$(электронный спин $S=3 / 2$ ), $\mathrm{Fe}^{2+}(S=2)$ и $\mathrm{Fe}^{3+}(S=5 / 2)$.
При исследовании кристаллов $\mathrm{ZnSe}$ : Cr было установлено, что зарядовое состояние ионов $\mathrm{Cr}$ существенно зависит от наличия и концентрации донорных и акцепторных уровней в кристалле, образованных примесью или собственными точечными дефектами нестехиометрии [2]. В частности, наличие донорных уровней переводит ионы $\mathrm{Cr}^{2+}$ в неактивное состояние $\mathrm{Cr}^{+}$, что существенно сказывается на характеристиках лазеров с использованием кристаллов $\mathrm{ZnSe}: \mathrm{Cr}$. Влияние концентрации нестехиометрических дефектов на концентрацию $\mathrm{Fe}^{2+}$ было обнаружено также в кристаллах $\mathrm{CdTe}: \mathrm{Fe}$ [3]. $\mathrm{B}$ обеих работах концентрация ионов $\mathrm{Fe}^{2+}$ контролировалась косвенно по спектрам оптического поглощения в ИК-области спектра. Более достоверную информацию о зарядовых состояниях примесных ионов можно получить методом ЭПР.

В работе [4] при исследовании кристаллов $\mathrm{ZnSe}: \mathrm{Fe}$ кроме спектра $\mathrm{Fe}^{2+}$ (переходы синглет-триплет на частоте $\sim 450 \mathrm{GHz}$ ) были обнаружены сигналы, соответствующие переходам между крамерсовыми дублетами моноклинных центров $\mathrm{Fe}^{3+}$. Низкая симметрия наблюдаемых центров, скорее всего, обусловлена локальной компенсацией избыточного заряда примесного иона. 
Таблица 1. Содержание 68 атомов Периодической таблицы элементов (в массовых процентах) в двух кристаллах ZnSe: Fe: K-900 и К-1003

\begin{tabular}{|c|c|c|c|c|c|}
\hline Атом & K-900 & K-1003 & Атом & K-900 & $\mathrm{K}-1003$ \\
\hline $\mathrm{Li}$ & $<3.29 \cdot 10^{-6}$ & $<2.72 \cdot 10^{-6}$ & In & $<9.50 \cdot 10^{-7}$ & $<7.85 \cdot 10^{-7}$ \\
\hline $\mathrm{Be}$ & $<7.18 \cdot 10^{-7}$ & $6.24 \cdot 10^{-7}$ & $\mathrm{Sn}$ & $<6.61 \cdot 10^{-7}$ & $4.39 \cdot 10^{-7}$ \\
\hline $\mathrm{B}$ & $<2.06 \cdot 10^{-6}$ & $<1.71 \cdot 10^{-6}$ & $\mathrm{Sb}$ & $<2.68 \cdot 10^{-7}$ & $<2.21 \cdot 10^{-7}$ \\
\hline $\mathrm{Na}$ & $<3.30 \cdot 10^{-6}$ & $<2.73 \cdot 10^{-6}$ & $\mathrm{Te}$ & $<6.45 \cdot 10^{-5}$ & $5.49 \cdot 10^{-5}$ \\
\hline $\mathrm{Mg}$ & $1.13 \cdot 10^{-5}$ & $3.49 \cdot 10^{-6}$ & $\mathrm{Cs}$ & $<1.64 \cdot 10^{-7}$ & $<1.36 \cdot 10^{-7}$ \\
\hline $\mathrm{Al}$ & $2.49 \cdot 10^{-6}$ & $<1.63 \cdot 10^{-5}$ & $\mathrm{Ba}$ & $3.17 \cdot 10^{-7}$ & $1.27 \cdot 10^{-7}$ \\
\hline $\mathrm{Si}$ & $<1.15 \cdot 10^{-4}$ & $<9.52 \cdot 10^{-5}$ & $\mathrm{La}$ & $1.01 \cdot 10^{-8}$ & $8.31 \cdot 10^{-9}$ \\
\hline $\mathrm{K}$ & $<1.74 \cdot 10^{-4}$ & $<1.44 \cdot 10^{-4}$ & $\mathrm{Ce}$ & $<3.77 \cdot 10^{-8}$ & $6.13 \cdot 10^{-8}$ \\
\hline $\mathrm{Ca}$ & $<5.13 \cdot 10^{-5}$ & $<4.24 \cdot 10^{-5}$ & $\operatorname{Pr}$ & $7.54 \cdot 10^{-9}$ & $2.49 \cdot 10^{-8}$ \\
\hline $\mathrm{Sc}$ & $<3.77 \cdot 10^{-9}$ & $<3.12 \cdot 10^{-9}$ & $\mathrm{Nd}$ & $<3.77 \cdot 10^{-9}$ & $<3.12 \cdot 10^{-9}$ \\
\hline $\mathrm{Ti}$ & $7.72 \cdot 10^{-7}$ & $1.19 \cdot 10^{-7}$ & $\mathrm{Sm}$ & $<3.77 \cdot 10^{-9}$ & $7.27 \cdot 10^{-8}$ \\
\hline V & $<2.11 \cdot 10^{-7}$ & $<1.75 \cdot 10^{-7}$ & $\mathrm{Eu}$ & $<2.18 \cdot 10^{-8}$ & $<1.80 \cdot 10^{-8}$ \\
\hline $\mathrm{Cr}$ & $2.39 \cdot 10^{-6}$ & $1.19 \cdot 10^{-6}$ & Gd & $<1.92 \cdot 10^{-7}$ & $<1.59 \cdot 10^{-7}$ \\
\hline $\mathrm{Mn}$ & $8.93 \cdot 10^{-7}$ & $3.89 \cdot 10^{-7}$ & $\mathrm{~Tb}$ & $2.51 \cdot 10^{-8}$ & $1.25 \cdot 10^{-8}$ \\
\hline $\mathrm{Fe}$ & $2.98 \cdot 10^{-3}$ & $1.51 \cdot 10^{-4}$ & Dy & $3.02 \cdot 10^{-8}$ & $<3.12 \cdot 10^{-9}$ \\
\hline Co & $<2.73 \cdot 10^{-6}$ & $<2.26 \cdot 10^{-6}$ & Ho & $5.66 \cdot 10^{-9}$ & $4.68 \cdot 10^{-9}$ \\
\hline $\mathrm{Ni}$ & $9.93 \cdot 10^{-7}$ & $2.10 \cdot 10^{-6}$ & $\mathrm{Er}$ & $<3.77 \cdot 10^{-9}$ & $<3.12 \cdot 10^{-9}$ \\
\hline $\mathrm{Cu}$ & $4.75 \cdot 10^{-4}$ & $4.73 \cdot 10^{-4}$ & $\mathrm{Tm}$ & $<3.77 \cdot 10^{-9}$ & $<3.12 \cdot 10^{-9}$ \\
\hline $\mathrm{Zn}$ & matrix & matrix & $\mathrm{Yb}$ & $<3.77 \cdot 10^{-9}$ & $<3.12 \cdot 10^{-9}$ \\
\hline $\mathrm{Ga}$ & $9.66 \cdot 10^{-6}$ & $8.93 \cdot 10^{-6}$ & $\mathrm{Lu}$ & $<1.85 \cdot 10^{-8}$ & $<1.53 \cdot 10^{-8}$ \\
\hline $\mathrm{Ge}$ & $4.64 \cdot 10^{-6}$ & $1.27 \cdot 10^{-6}$ & $\mathrm{Hf}$ & $<3.77 \cdot 10^{-9}$ & $<3.12 \cdot 10^{-9}$ \\
\hline As & $2.16 \cdot 10^{-5}$ & $1.97 \cdot 10^{-5}$ & $\mathrm{Ta}$ & $<3.77 \cdot 10^{-9}$ & $<3.12 \cdot 10^{-9}$ \\
\hline $\mathrm{Se}$ & matrix & matrix & W & $1.05 \cdot 10^{-6}$ & $1.00 \cdot 10^{-7}$ \\
\hline $\mathrm{Rb}$ & $3.71 \cdot 10^{-8}$ & $<4.51 \cdot 10^{-7}$ & $\operatorname{Re}$ & $<3.77 \cdot 10^{-9}$ & $1.87 \cdot 10^{-8}$ \\
\hline $\mathrm{Sr}$ & $<2.87 \cdot 10^{-7}$ & $<2.37 \cdot 10^{-7}$ & Os & $1.84 \cdot 10^{-7}$ & $1.87 \cdot 10^{-8}$ \\
\hline $\mathrm{Y}$ & $<1.51 \cdot 10^{-7}$ & $<1.25 \cdot 10^{-7}$ & $\mathrm{Ir}$ & $<5.81 \cdot 10^{-8}$ & $<4.80 \cdot 10^{-8}$ \\
\hline $\mathrm{Zr}$ & $4.80 \cdot 10^{-6}$ & $1.68 \cdot 10^{-6}$ & $\mathrm{Pt}$ & $1.12 \cdot 10^{-7}$ & $2.46 \cdot 10^{-7}$ \\
\hline $\mathrm{Nb}$ & $2.78 \cdot 10^{-5}$ & $2.45 \cdot 10^{-5}$ & $\mathrm{Au}$ & $5.09 \cdot 10^{-8}$ & $4.21 \cdot 10^{-8}$ \\
\hline Mo & $<1.84 \cdot 10^{-6}$ & $4.96 \cdot 10^{-6}$ & $\mathrm{Hg}$ & $9.36 \cdot 10^{-7}$ & $6.82 \cdot 10^{-7}$ \\
\hline $\mathrm{Ru}$ & $<4.15 \cdot 10^{-7}$ & $<3.43 \cdot 10^{-7}$ & $\mathrm{Tl}$ & $<8.71 \cdot 10^{-9}$ & $4.16 \cdot 10^{-9}$ \\
\hline $\mathrm{Rh}$ & $6.28 \cdot 10^{-8}$ & $1.25 \cdot 10^{-8}$ & $\mathrm{~Pb}$ & $1.28 \cdot 10^{-7}$ & $4.52 \cdot 10^{-8}$ \\
\hline $\mathrm{Pd}$ & $9.68 \cdot 10^{-8}$ & $<1.87 \cdot 10^{-8}$ & $\mathrm{Bi}$ & $4.65 \cdot 10^{-8}$ & $7.27 \cdot 10^{-9}$ \\
\hline $\mathrm{Ag}$ & $2.26 \cdot 10^{-7}$ & $3.26 \cdot 10^{-7}$ & Th & $<3.77 \cdot 10^{-9}$ & $<3.12 \cdot 10^{-9}$ \\
\hline $\mathrm{Cd}$ & $9.62 \cdot 10^{-6}$ & $4.15 \cdot 10^{-6}$ & $\mathrm{U}$ & $1.26 \cdot 10^{-8}$ & $<3.12 \cdot 10^{-9}$ \\
\hline
\end{tabular}

Данная работа посвящена детальному исследованию методом магнитного резонанса точечных дефектов в монокристаллах $\mathrm{ZnSe}$ с примесью железа.

\section{2. Методика измерений}

Кристаллы селенида цинка выращивались в кварцевой ампуле из паровой фазы методом физического транспорта на затравку ZnSe в атмосфере гелия [5]. В качестве исходных материалов использовался поликристаллический $\mathrm{ZnSe}$, полученный методом химического осаждения из пара в Институте химии высокочистых веществ им. Г.Г. Девятых РАН, и поликристаллический $\mathrm{FeSe}$, синтезированный из особо чистого $\mathrm{Fe}$ компании Merck и особо чистого Se завода „Красный химик“. Типичный состав примесей двух кристаллов $\mathrm{ZnSe}: \mathrm{Fe} \mathrm{c} \mathrm{большей}\left(\sim 2 \cdot 10^{18} \mathrm{~cm}^{-3}, \mathrm{~K}-900\right)$ и меньшей $\left(\sim 10^{17} \mathrm{~cm}^{-3}\right.$, К-1003) концентрацией ионов Fе представлен в табл. 1. Анализ содержания примесей был выполнен в Российском химико-технологическом университете им. Д.И. Менделеева. Для анализа использовался метод масс-спектроскопии с индуктивно-связанной плазмой на современном оборудовании (NexION 300D, Perkin Elmer Inc.). Видно, что из неконтролируемых примесей наибольшую массовую концентрацию имеет примесь $\mathrm{Cu}\left(4.7 \cdot 10^{-4}\right.$ mass\%). Кроме того, сравнимые $\mathrm{c}$ Fe концентрации могут иметь ионы кислорода (не представлена в табл. 1) и дефекты нестехиометрии (в основном $V_{\mathrm{Zn}}$ ).

Исследовался кристалл $\mathrm{ZnSe}$ с концентрацией железа примерно $10^{18} \mathrm{~cm}^{-3}$. Все образцы были вырезаны из одной були К-900. Образец № 1 имел форму куба с размером ребра около $7.5 \mathrm{~mm}$ с гранями нормальными кристаллическим осям $C_{2}$ и $C_{4}$. Для помещения образца в резонатор спектрометра (диаметр отверстия $10 \mathrm{~mm}$ ) 
вертикальные (параллельные осям $C_{2}$ ) ребра были слегка сошлифованы. Образцы № 2 и 3 имели размеры $\sim 8 \times 4.5 \times 4 \mathrm{~mm}$ и $\sim 6 \times 3 \times 2.5 \mathrm{~mm}$ соответственно, оба с направлением $\langle 110\rangle$ вдоль длинной оси.

Для измерений в диапазоне частот $(37-850 \mathrm{GHz})$ использовался широкополосный ЭПР-спектрометр, созданный на основе генераторов микроволнового излучения - ламп обратной волны. Измерения на нем выполнялись при температуре жидкого гелия в магнитных полях до $900 \mathrm{mT}$. Измерения спектров парамагнитного резонанса при комнатной температуре и $110 \mathrm{~K}$ проводились на спектрометре трехсантиметрового диапазона EMX Plus Bruker в полях до $1450 \mathrm{mT}$.

Для получения угловых зависимостей образец № 1 приклеивался к торцу стеклянной трубки, закрепленной в штанге штатного одноосного автоматического гониометра. Образец № 2 помещался в фторопластовый бочонок, вращающийся в вертикальной плоскости и расположенный на штанге гониометра, что позволяло при комнатной температуре с большей точностью получить ориентации: $\mathbf{B}\left\|\mathbf{C}_{2}, \mathbf{B}\right\| \mathbf{C}_{3}, \mathbf{B} \| \mathbf{C}_{4}$ (где $\mathbf{B}-$ индукция магнитного поля, $C_{i}-$ оси симметрии кристалла). Измерения при пониженной температуре проводились на образце № 3, помещенном внутрь штатной (ER221TUB/4) кварцевой ампулы, и № 2, приклеенном к ее торцу.

\section{3. Результаты эксперимента}

Исследуемые кристаллы ZnSe имеют структуру кубического сфалерита (цинковой обманки) с пространственной группой $T_{d}^{2}(F \overline{4} 3 m)$ и параметром элементарной ячейки $5.66 \AA$. По расположению атомов она подобна структуре алмаза, однако из-за наличия атомов двух сортов не имеет центра инверсии. Это гранецентрированная кубическая решетка, в которой заселена половина тетраэдрических пустот.

Проведенные нами измерения частотно-полевых зависимостей междублетных переходов моноклинных центров $\mathrm{Fe}^{3+}$ (рис. 1), обнаруженных в [4], дали для расщеплений в нулевом магнитном поле следующие величины: $124( \pm 1 / 2 \leftrightarrow \pm 3 / 2)$ и $203 \mathrm{GHz}( \pm 3 / 2 \leftrightarrow \pm 5 / 2)$.

С целью определения параметров спинового гамильтониана моноклинных центров $\mathrm{Fe}^{3+}$ в $\mathrm{ZnSe}$ было проведено детальное исследование ориентационного поведения ЭПР спектра образца № 1 на частоте $9156 \mathrm{MHz}$ при вращении магнитного поля в плоскости $(110)$ с шагом $2^{\circ}$ при комнатной температуре. Результаты приведены на рис. 2. Как хорошо видно, вблизи $g=2$ наблюдается сигналы сверхтонкой структуры кубических центров $\mathrm{Mn}^{2+}$, исследованных авторами [6]. Остальные интенсивные сигналы можно поделить на две группы: первая узкие сигналы $(\sim 2 \mathrm{mT})$, наблюдаемые при $\mathbf{B} \| C_{2}$ в полях 164 и $230 \mathrm{mT}$, вторая - более широкие $(\sim 5 \mathrm{mT})$, наблюдаемые в диапазоне 70-440 mT.

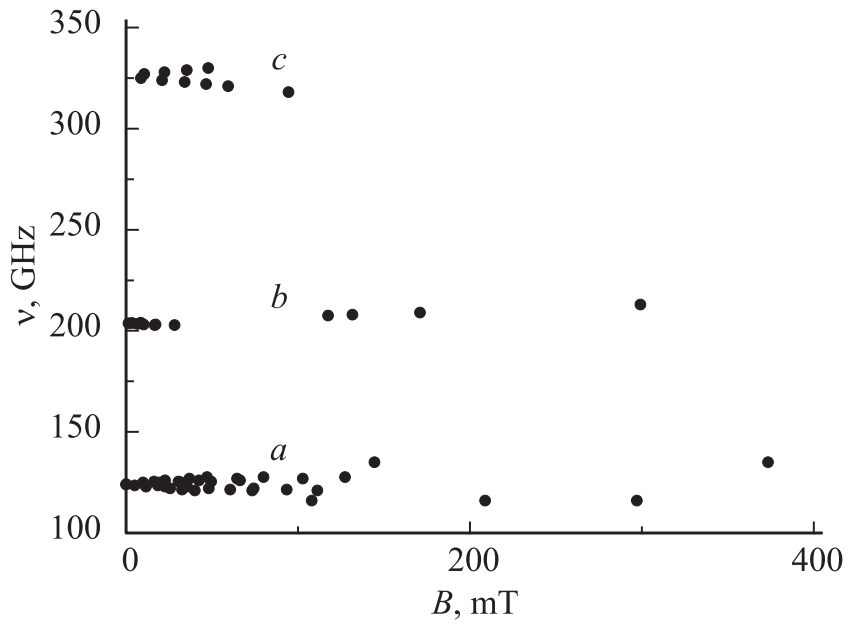

Рис. 1. Частотно-полевые зависимости междублетных резонансных переходов иона $\mathrm{Fe}^{3+}$ в кристалле $\mathrm{ZnSe}$ (образец № 1) при $4.2 \mathrm{~K}$ в произвольной ориентации магнитного поля: $a-$ переходы $\pm 1 / 2 \leftrightarrow \pm 3 / 2 ; b-$ переходы $\pm 3 / 2 \leftrightarrow \pm 5 / 2$; $c$ - переходы $\pm 1 / 2 \leftrightarrow \pm 5 / 2$.

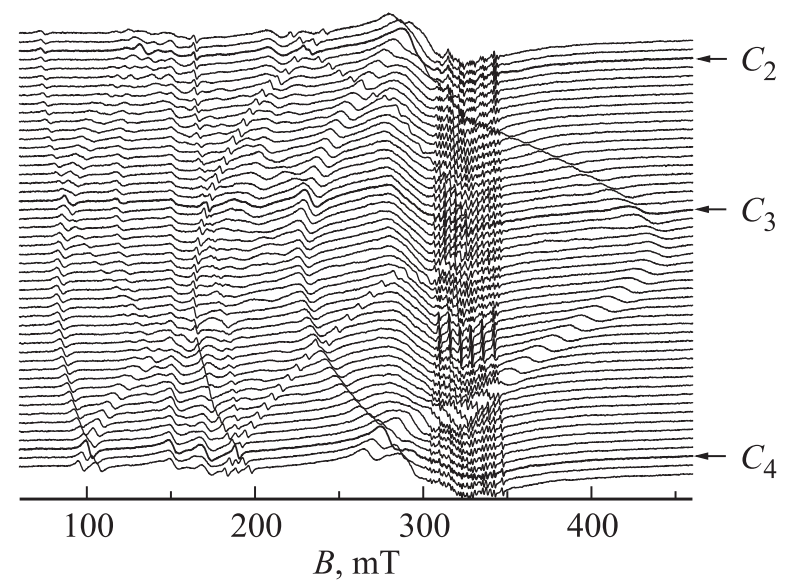

Рис. 2. Ориентационное поведение спектра ЭПР (образец № 1) при комнатной температуре на частоте $9156 \mathrm{MHz}$ при вращении магнитного поля в плоскости (110).

3.1. При анализе угловых зависимостей второй группы сигналов было замечено, что они качественно аналогичны зависимостям моноклинного дипольного комплекca $\mathrm{Fe}^{3+}-\mathrm{Cu}^{+}$в $\mathrm{ZnSe}[7,8]$, в котором $\mathrm{Fe}^{3+}$ замещает ион $\mathrm{Zn}^{2+}$ и содержит в ближайшей катионной позиции (в направлении $\langle 110\rangle$ на расстоянии $0.4 \mathrm{~nm}$ ) ион $\mathrm{Cu}^{+}$. Исследованные в работах $[7,8]$ кристаллы $\mathrm{ZnSe}$, легированные железом и медью, имеют 12 одинаковых, но различно ориентированных $\mathrm{Fe}^{3+}-\mathrm{Cu}^{+}$комплексов с симметрией $C_{S}$. В работе [7] наблюдались сигналы указанных центров только в нижнем $( \pm 1 / 2)$ крамерсовом дублете; авторам [8] удалось детектировать переходы как в нижнем, так и в среднем $( \pm 3 / 2)$ дублетах.

Описание спектра наблюдаемых нами моноклинных центров $\mathrm{Fe}^{3+}$ проводилось спиновым гамильтонианом в 
Таблица 2. Параметры спинового гамильтониана дипольных центров $\mathrm{Fe}^{3+}-\mathrm{Cu}^{+}$и моноклинных центров $\mathrm{Fe}^{3+}$ в $\mathrm{ZnSe}$

\begin{tabular}{l|c|c|c}
\hline Параметры & \multicolumn{2}{|c|}{$\begin{array}{c}\text { Дипольный центр } \\
\mathrm{Fe}^{3+}-\mathrm{Cu}^{+}[7]\end{array}$} & $\begin{array}{c}\text { Моноклинный центр } \mathrm{Fe}^{3+} \\
\text { [данная работа] }\end{array}$ \\
\hline$g_{\|}$ & 2.058 & 2.058 & 2.043 \\
$g_{\perp}$ & 2.058 & 2.058 & 2.062 \\
$b_{20}$ & $41281^{*}$ & $40567^{*}$ & $51720(15)^{*}$ \\
$b_{21}$ & 0 & 24361 & $31150(150)$ \\
$b_{22}$ & 17160 & 17865 & $23710(15)$ \\
$b_{40}$ & - & - & $110(20)$ \\
$F(N)$ & - & - & $62(87)$ \\
$\tau$, degree $^{* *}$ & 6.6 & 0 & 0
\end{tabular}

Примечание. $b_{n m}$ и среднеквадратичное отклонение расчетных частот от экспериментальных $F(N)$ в $\mathrm{MHz}, N-$ число используемых в оптимизации положений переходов.

* Предполагается, что $b_{20}>0$.

** При $\tau=0^{\circ} \mathbf{Z} \| C_{3}$ и $b_{21} \neq 0\left(\tau-\right.$ угол между $C_{3}$ и $\left.\mathbf{Z}\right)$.

определении [9]

$$
\begin{aligned}
& H_{s p}=g \beta(\mathbf{B S})+1 / 3\left(b_{20} O_{20}+b_{21} O_{21}+b_{22} O_{22}\right) \\
& +1 / 60\left(b_{40} O_{40}+b_{41} O_{41}+b_{42} O_{42}+b_{43} O_{43}+b_{44} O_{44}\right),
\end{aligned}
$$

где $g-g$-фактор, $\beta-$ магнетон Бора, $O_{n m}-$ спиновые операторы Стивенса [9], $b_{n m}$ - параметры тонкой структуры. Использовалась следующая локальная система координат: $\mathbf{Z}$ параллельна оси $C_{3}$ кристалла, лежащей в плоскости отражения $\sigma$ рассматриваемого комплекса, $\mathbf{Y} \perp \sigma$, ось $\mathbf{X}$, естественно, располагается тоже в плоскости $\sigma$. Система координат работы [7] повернута относительно нашей на угол $\tau$ вокруг оси $\mathbf{Y}$, в результате чего параметр $b_{21}$ в (1) обращается в нуль (табл. 2).

Оптимизация с учетом 63 положений внутридублетных переходов $(-1 / 2 \leftrightarrow+1 / 2$ и $-3 / 2 \leftrightarrow+3 / 2)$, показанных на рис. 2 и 3 , а также величин начальных расщеплений $(124$ и $203 \mathrm{GHz})$, показала совместимость результатов высокочастотных и низкочастотных измерений и привела к следующим параметрам спинового гамильтониана (1)

$$
\begin{gathered}
g=2.058, \quad b_{20}=51600, \quad b_{21}=32320, \\
b_{22}=23720, \quad b_{40}=150
\end{gathered}
$$

(где все $b_{n m}$ в MHz). Остальные параметры четвертого ранга оказались порядка погрешности их определения и поэтому не приводятся.

Включение в процедуру оптимизации ориентационного поведения положений сигналов в плоскости (111) на частоте $125.7 \mathrm{GHz}$ (рис. 4) и допущение анизотропии $g$-фактора несколько изменило параметры. Их значения и среднеквадратичное отклонение в сравнении с результатами [7] для комплексов $\mathrm{Fe}^{3+}-\mathrm{Cu}^{+}$приведены в табл. 2. Большая величина среднеквадратичного

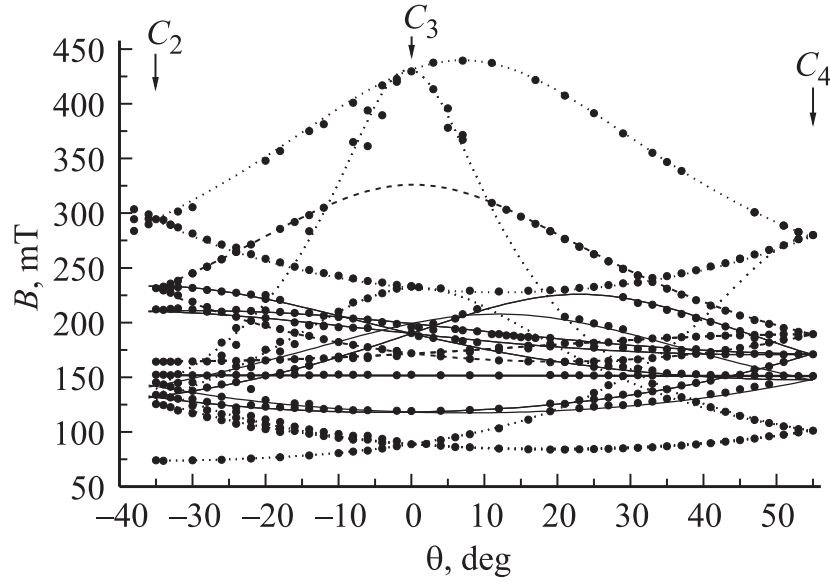

Рис. 3. Ориентационное поведение экспериментальных (образец №1) и расчетных положений сигналов при вращении магнитного поля в плоскости (110) на частоте $9156 \mathrm{MHz}$ Сплошные кривые - переходы моноклинных центров $\mathrm{Fe}^{3+}$ в среднем дублете $( \pm 3 / 2)$, пунктирные - в нижнем дублете $( \pm 1 / 2)$. Штриховые кривые - переходы тригональных центров со спином $3 / 2$ в нижнем дублете $( \pm 1 / 2)$. Жирная прямая - сигнал с $g=4.3$.

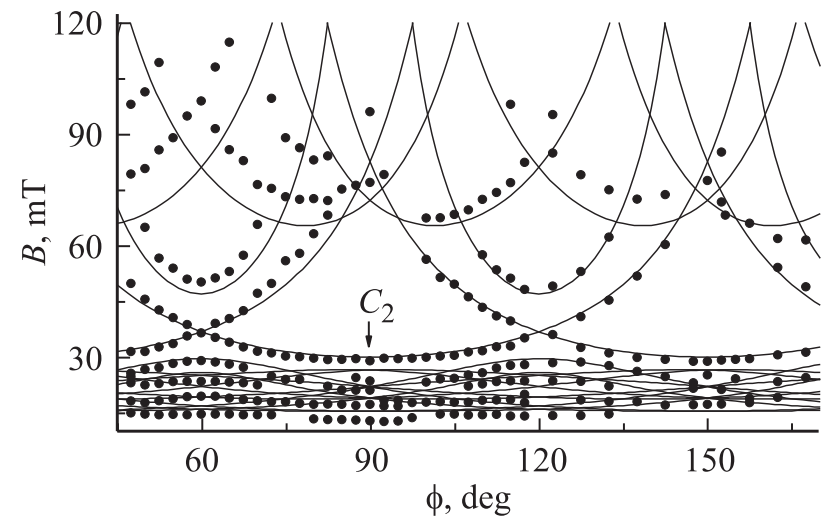

Рис. 4. Угловая зависимость положений переходов моноклинных центров $\mathrm{Fe}^{3+}$ на частоте $125.7 \mathrm{GHz}$ при вращении магнитного поля в плоскости (111): точки - эксперимент, кривые - расчет.

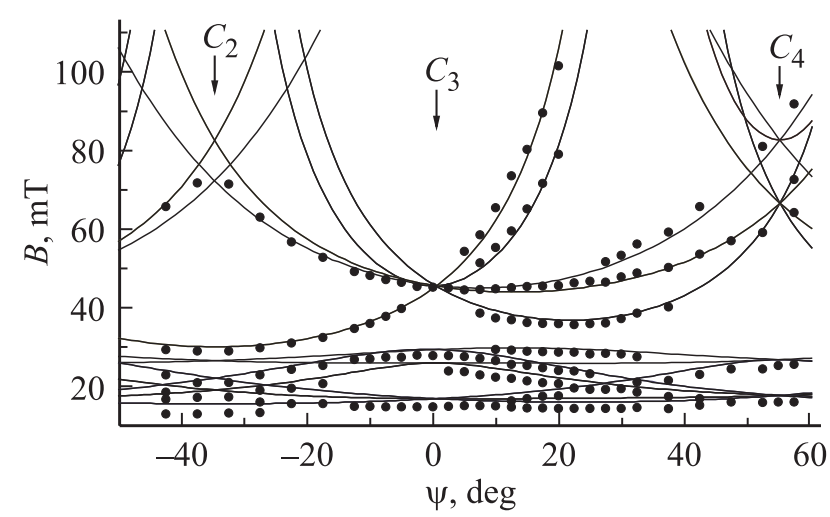

Рис. 5. Ориентационное поведение экспериментальных и расчетных положений сигналов моноклинных центров $\mathrm{Fe}^{3+}$ при вращении магнитного поля в плоскости (110) на частоте 125.7 GHz. 
отклонения $F$ расчетных частот от экспериментальных обусловлена использованием положений переходов, полученных при разных температурах, а также погрешностью в определении частоты $( \pm 0.5 \mathrm{GHz})$ в диапазоне 70-350 GHz. Рис. 5 демонстрирует описание гамильтонианом (1) с параметрами табл. 2 угловой зависимости положений сигналов на частоте $125.7 \mathrm{GHz}$ в плоскости (110).

3.2. Первую группу сигналов (см. выше) можно объяснить переходами в нижнем крамерсовом дублете $( \pm 1 / 2$, предполагается, что $\left.b_{20}>0\right)$ тригональных центров со спином $S=3 / 2$, большим начальным расщеплением и $g_{\|} \approx g_{\perp} \approx 2$. Оптимизация со спиновым гамильтонианом вида [9] при $z \| C_{3}$ (где $C_{3}$ операция симметрии центра)

$$
H_{s p}=g \beta(\mathbf{B S})+1 / 3 b_{20} O_{20}
$$

и положениями сигналов, показанными на рис. 3 , дали параметры $g=2.006, b_{20}=37 \mathrm{GHz}$.

Такой гамильтониан предсказывает при $\mathbf{B} \| C_{2}$ для двух (из четырех) магнитно эквивалентных центров, имеющих полярный угол $\theta=90^{\circ}$, максимум положения перехода в верхнем крамерсовом дублете $( \pm 3 / 2)$ в поле $\sim 1300 \mathrm{mT}$. В этом районе были зарегистрированы слабые ЭПР-сигналы с ориентационным поведением, показанным на рис. 6. Наблюдаемую картину (неэквивалентность двух центров) можно объяснить, допуская, что при измерении угловой зависимости магнитное поле в районе $C_{2}$ отклонилось от плоскости (110) на угол $\sim 2.2^{\circ}$. Такая погрешность в ориентации образца вполне возможна.

Ориентацию $\theta=90^{\circ}$ для любого из тригональных центров можно получить также поворотом на $90^{\circ}$ от $\mathbf{B} \| C_{3}$ (ось $C_{3}$ этого центра) в плоскости (110). Реализация нами указанной ориентации и наблюдение в ней сигнала в поле 1300 тТ является подтверждением существования именно тригонального (аксиального) центра с $S=3 / 2$.

Включение в процедуру оптимизации переходов в верхнем дублете $( \pm 3 / 2)$ привело к следующим параметрам: $g_{\|}=2.00(1), g_{\perp}=1.99(1), b_{20}=40.5(5) \mathrm{GHz}$ со среднеквадратичным отклонением $30 \mathrm{MHz}$ при учете 36 резонансных положений. Расчетные угловые зависимости с этими параметрами приведены на рис. 3 и 6.

Следует заметить, что попытка описать наблюдаемые угловые зависимости положений переходов в двух крамерсовых дублетах спиновым гамильтонианом с $S=5 / 2$, включающем кроме (2) слагаемое

$$
1 / 60\left(b_{40} O_{40}+b_{43} O_{43}\right)
$$

окончилась неудачей. Среднеквадратичное отклонение в процедуре оптимизации (с $g=2$, характерным для $\mathrm{Fe}^{3+}$ ) возрастало на порядок, аномально росли значения $b_{20}$ и $b_{43}$. При варьировании $g$-тензора его $x, y$-компоненты стремились к значению 1.3, что абсолютно невероятно для центров $\mathrm{Fe}^{3+}$.

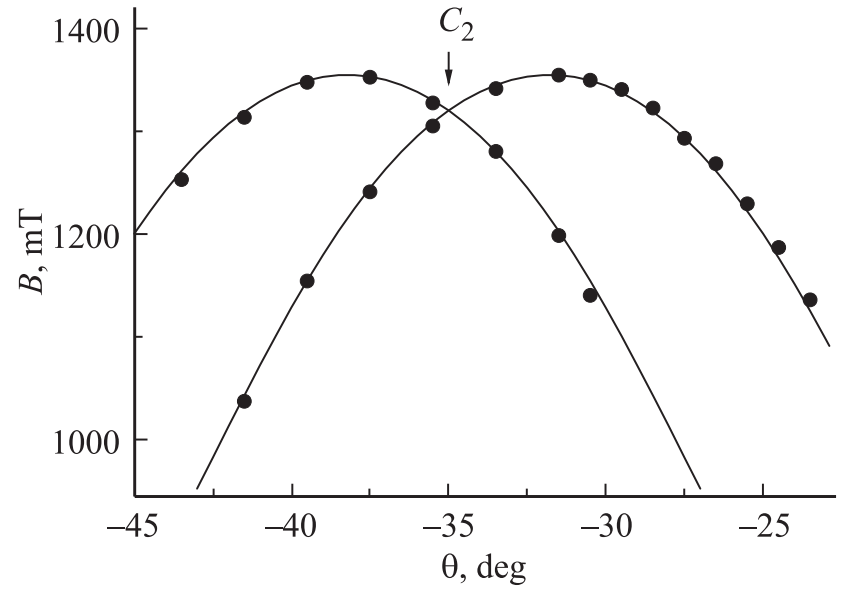

Рис. 6. Угловая зависимость положений переходов в верхнем дублете тригональных центров со спином 3/2 при вращении магнитного поля в плоскости (110) на частоте $9156 \mathrm{MHz}$ Точки - эксперимент, кривые - расчет для случая, когда магнитное поле отстоит от плоскости $(110)$ в районе $C_{2}$ на угол $2.2^{\circ}$.

Поскольку вычисленное нулевое расщепление между двумя дублетами тригонального центра с $S=3 / 2$ в данном случае равно $2 b_{20}=81 \mathrm{GHz}$, мы осуществили поиск междублетных переходов в этой области частот. Хотя тригональный центр обнаружен не был, регистрировались резонансные переходы типа $0 \leftrightarrow \pm 1$ иона $\mathrm{Cr}^{2+}$. ЭПР-спектроскопия иона $\mathrm{Cr}^{2+}\left(d^{4},{ }^{5} D\right.$, $S=2)$ в $\mathrm{ZnSe}$ хорошо изучена [10-12], и идентификация этой примеси не представляет сложности. Во-первых, угловая зависимость обнаруженных переходов (в статье не показана) свидетельствовала о тетрагональной симметрии центров, что соответствует литературным данным. Во-вторых, должны были наблюдаться резонансные переходы типа $\pm 1 \leftrightarrow \pm 2$. Энергия в нулевом магнитном поле пропорциональна квадрату спиновой проекции, поэтому отношение энергетических зазоров между спиновыми состояниями равно $1: 3$. Предприняв поиск сигналов на частотах в 3 раза больших, мы обнаружили соответствующие линии ЭПР. На рис. 7 представлены спектры ЭПР, измеренные вблизи двух расщеплений в нулевом поле в исследуемом кристалле. Причем большое количество линий для переходов $\pm 1 \leftrightarrow \pm 2$ объясняется тем, что регистрировались линии от нескольких магнитно неэквивалентных центров как для разрешенных, так и для запрещенных переходов.

Тот факт, что в окрестности $81 \mathrm{GHz}$ не удалось детектировать междублетные переходы тригональных центров, может быть обусловлен большой погрешностью в определении величины начального расщепления в результате оптимизации с учетом положений только внутридублетных переходов (см. разд. 3.1).

Во всех исследованных ориентациях образца № 1 наблюдался ЭПР-сигнал с $g \approx 4.3$ (рис. 2, 3). Аналогичные сигналы детектировались в стеклах, поли- и 

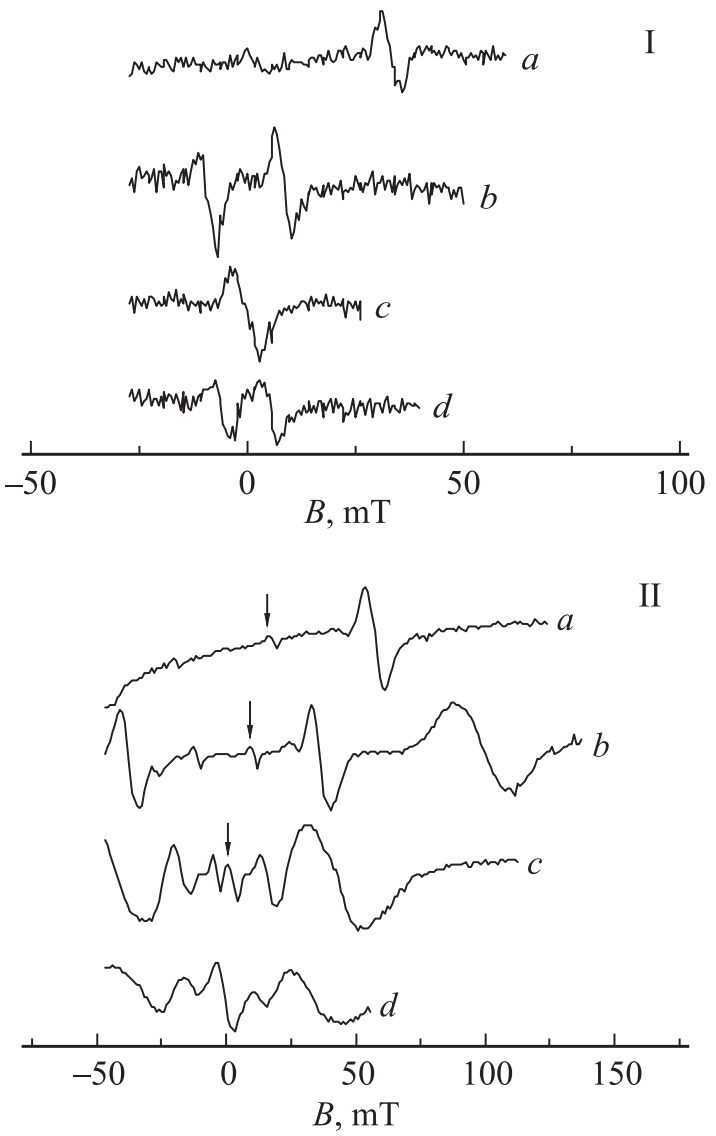

Рис. 7. Вид спектров ЭПР иона $\mathrm{Cr}^{2+}$ в кристалле $\mathrm{ZnSe}$ вблизи расщеплений в нулевом поле (произвольная ориентация магнитного поля). I - переходы типа $0 \leftrightarrow \pm 1$, частоты: $a-75 \mathrm{GHz}, b-74.5 \mathrm{GHz}, c-74.4 \mathrm{GHz}, d-74.3 \mathrm{GHz}$. II - переходы типа $\pm 1 \leftrightarrow \pm 2$, частоты: $a-225 \mathrm{GHz}$, $b-224.5 \mathrm{GHz}, c-224 \mathrm{GHz}, d-223.9 \mathrm{GHz}$. Стрелками показаны линии запрещенных переходов с $\Delta m_{s}=3$.

монокристаллах с примесью железа в работах [13-15]. Авторами $[15,16]$ было показано, что указанный изотропный сигнал обусловлен переходом в среднем крамерсовом дублете ромбических центров $\mathrm{Fe}^{3+}$ при $b_{20} \gg g \beta B$ и равенстве величин $b_{20}=b_{22}$ (в повернутой на $90^{\circ}$ системе координат $b_{20}=0$ ), а также малых параметрах четвертого ранга. Поскольку в других образцах $\mathrm{ZnSe}: \mathrm{Fe}$ (№ 2 и 3) указанный сигнал на наблюдается, скорее всего, он обусловлен парамагнитными центрами в стеклянной трубке и склейке, на которых был подвешен образец.

\section{4. Обсуждение результатов}

Предполагается, что в исследуемом кристалле заметная концентрация ионов меди маловероятна, поэтому наблюдаемые моноклинные центры (вторые) трехзарядного железа нами были отнесены к ассоциатам типа ион $\mathrm{Fe}^{3+}$ - вакансия ближайшего катиона в направлении $\langle 110\rangle$. Однако, когда описанная работа была сделана, мы получили информацию о содержании в исследованных образцах 68 различных примесей (табл. 1). В связи с тем, что концентрации железа и меди в кристаллах оказались, как и в $[7,8]$, сравнимы, нами были проведены измерения формы сигналов при $110 \mathrm{~K}$. Наблюдаемая квартетная структура низкополевых сигналов моноклинных центров $\mathrm{Fe}^{3+}$ (рис. 8) убедительно доказывает, что зарядовая компенсация $\mathrm{Fe}^{3+}$ осуществляется ионами $\mathrm{Cu}^{+}$. Расщепление указанных ЭПР-сигналов обусловлено сверхтонким взаимодействием с ядерными спинами меди $\left({ }^{63} \mathrm{Cu}, I=3 / 2\right.$, распространенность $69 \%,{ }^{65} \mathrm{Cu}$, $I=3 / 2,31 \%)$.

Следует заметить, что отношения приведенных в табл. 2 величин параметров $b_{20}, b_{21}, b_{22}$, определенных нами и авторами $[7,8]$, практически одинаковы и примерно равны 1.3. Это обусловлено тем, что измерения на одной микроволновой частоте положений только внутридублетных переходов, что было сделано в $[7,8]$, дает достоверную информацию о соотношении параметров $b_{2 m}$, тогда как их абсолютные значения слабо зависят от величины начальных расщеплений и поэтому определяются с большой погрешностью. В связи с этим трудно надеяться, что абсолютные значения параметров в $[7,8]$ определены достаточно хорошо. Действительно, расчет с параметрами спинового гамильтониана, полученными
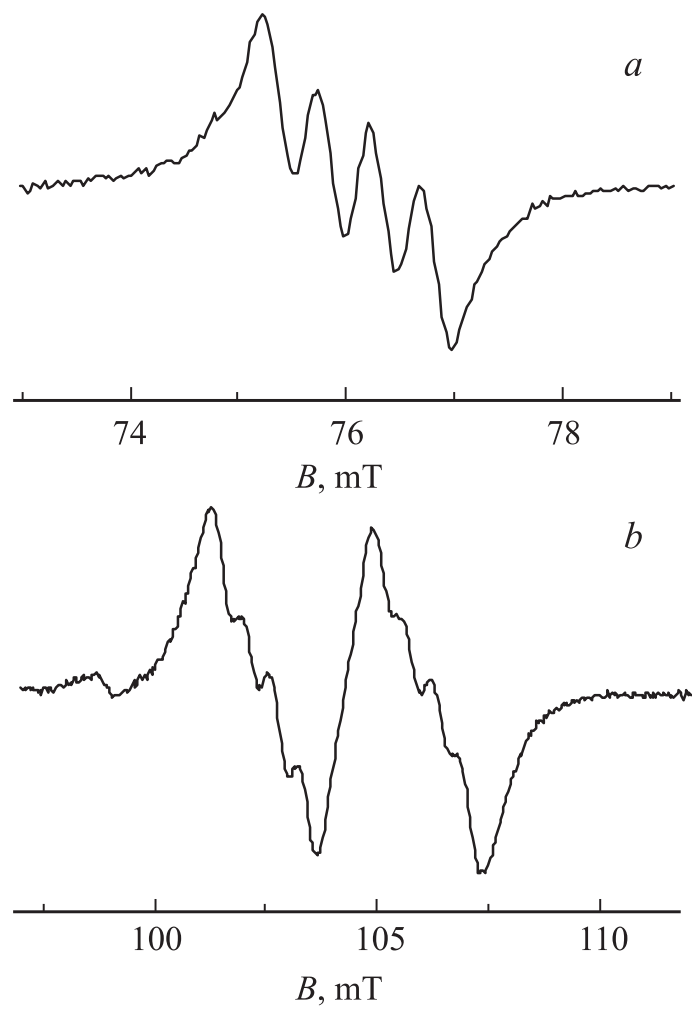

Рис. 8. Форма сигналов низкополевых переходов моноклинных центров $\mathrm{Fe}^{3+}$ при $\mathbf{B}\left\|C_{2}(a), \mathbf{B}\right\| C_{4}(b)$ и $110 \mathrm{~K}$ на частоте $9387 \mathrm{MHz}$ (образец № 2). Два сигнала, детектируемые в ориентации $\mathbf{B} \| C_{4}(b)$, при комнатной температуре (см. рис. 2, 3 ) не разрешаются. 
Таблица 3. Параметры спинового гамильтониана тетрагональных центров $\mathrm{Fe}^{+}$в кубических кристаллах

\begin{tabular}{c|c|c|c}
\hline Параметры & $\mathrm{ZnS}[17]$ & $\mathrm{ThO}_{2}[18]$ & $\mathrm{SrCl}_{2}[19,20]$ \\
\hline$T$ & $1.3 \mathrm{~K}$ & $77 \mathrm{~K}$ & $4 \mathrm{~K}$ \\
$g_{\|}$ & 2.251 & 2.0117 & 2.000 \\
$g_{\perp}$ & 2.251 & 2.0630 & 4.174 \\
$b_{20}=D, \mathrm{GHz}$ & - & 88.4 & 121.5
\end{tabular}

в [7,8], дает для расщеплений в нулевом магнитном поле величины 97 и $160 \mathrm{GHz}$, которые заметно отличаются от измеренных. Таким образом, нами установлено, что моноклинные центры трехзарядного железа являются ассоциатами $\mathrm{Fe}^{3+}$ - ион $\mathrm{Cu}^{+}$, заместившими ближайший ион $\mathrm{Zn}^{2+}$, при этом получены более адекватные величины параметров спинового гамильтониана.

Обнаруженные и исследованные нами в ZnSe тригональные центры $(S=3 / 2)$ могут быть обусловлены ионами группы железа $\mathrm{Fe}^{+}, \mathrm{Ni}^{3+}, \mathrm{Cr}^{3+}$, имеющими спин $3 / 2$ и не обладающими протяженной сверхтонкой структурой, превышающей наблюдаемую ширину сигналов. О присутствии ионов хрома и никеля в $\mathrm{ZnSe}$, легированном железом, свидетельствуют данные табл. 1; о возможности их наблюдения говорит успешное детектирование спектров ионов $\mathrm{Cr}^{2+}$ и $\mathrm{Mn}^{2+}$ (см. разд. 3), концентрация которых примерно такая же (табл. 1). Кроме того, наблюдаемые тригональные центры могут быть связаны с дефектами более сложной структуры (ассоциатами, парами и т.д.).

Спектр ЭПР тетрагональных центров $\mathrm{Fe}^{+}$с локальной компенсацией заряда исследован в кубических кристаллах $\mathrm{ZnS}, \mathrm{ThO}_{2}$ и $\mathrm{SrCl}_{2}$ авторами [17-20]. Наблюдаемые ими спектры обусловлены переходами внутри спинового квартета орбитального синглета $\Gamma_{2}$, возникающего в результате расщепления кубическим кристаллическим полем ${ }^{4} F$-терма свободного иона $\mathrm{Fe}^{+}$. Аналогичная ситуация может складываться для ионов $\mathrm{Fe}^{+}$и $\mathrm{Ni}^{3+}$ в $\mathrm{ZnSe}$ в случае их локальной зарядовой компенсации.

В сульфиде цинка и хлориде стронция центры $\mathrm{Fe}^{+}$возникали после ультрафиолетового [17] или рентгеновского $[19,20]$ облучения. Значения $g$-факторов и начального расщепления $\mathrm{Fe}^{+}$, полученные в [17-20], приведены в табл. 3. Авторами [18] наблюдалось сильное уширение ЭПР-сигналов $\mathrm{Fe}^{+}$-центров в $\mathrm{ThO}_{2}$ выше $100 \mathrm{~K}$, вызванное ускорением спин-решеточной релаксации.

Аксиальные центры $\mathrm{Ni}^{3+}(S=3 / 2)$ после ультрафиолетового облучения кристаллов $\mathrm{ZnO}$ (структура вюртцита), легированных никелем, были детектированы при 1.3 и $4.2 \mathrm{~K}$ авторами [17]. Получены следующие параметры спинового гамильтониана: $g=2.1426$, $b_{20}=D \approx 2 \mathrm{~cm}^{-1}$.

Тригональные центры $\mathrm{Cr}^{3+}$ и $\mathrm{V}^{2+}$ в кристаллах структуры флюорита были исследованы в работах [21,22] при температуре $77 \mathrm{~K}$. Для ионов $\mathrm{Cr}^{3+}$ получены параметры $g_{\|}=1.961, \quad g_{\perp}=1.97, \quad b_{20}=59.4 \mathrm{GHz} \quad$ в
$\mathrm{CaF}_{2}$ [21] и $g_{\|}=1.966, g_{\perp}=1.97, b_{20}=54.0 \mathrm{GHz}$ в $\mathrm{CdF}_{2}$ [22]. Обнаружено аномально большое сверхтонкое взаимодействие с двумя эквивалентными ядрами фтора, приводящее к появлению двух сателлитов основного ЭПР-сигнала. Авторы работы [21] считают, что причиной наблюдаемого тригонального искажения (сжатия куба из восьми ближайших ионов фтора вдоль пространственной диагонали) обоих центров является эффект Яна-Теллера, поскольку основным состоянием иона $\mathrm{Cr}^{3+}$ (как и $\mathrm{V}^{2+}$ ) в восьмикратном окружении будет орбитальный триплет $\Gamma_{4}$.

Расчет для иона $\mathrm{V}^{2+}$ эффекта Яна-Теллера в теории кристаллического поля, проведенный авторами [23], привел к заключению о растяжении фторового куба вдоль тригональной оси. В результате учета ковалентности связи парамагнитного иона $\mathrm{V}^{2+}$ (как и $\left.\mathrm{Cr}^{3+}\right)$ с лигандами [23] была показана возможность изменения знака искажения, т.е. смещения двух ионов фтора вдоль $C_{3}$ внутрь куба.

Как видно, величины измеренных нами параметров спинового гамильтониана (2) тригонального центра с $S=3 / 2$ не противоречат явно данным, полученным для ионов $\mathrm{Fe}^{+}, \mathrm{Ni}^{3+}, \mathrm{Cr}^{3+}$ в позициях с кубическим и тетраэдрическим окружением [17-23]. Из-за большой концентрации в кристаллах ионов железа (табл. 1) можно было отдать предпочтение центрам $\mathrm{Fe}^{+}$. Однако ионы железа со степенью окисления +1 нестабильны, об этом, в частности, свидетельствует получение авторами [17-20] центров $\mathrm{Fe}^{+}$путем ультрафиолетового или рентгеновского облучения. Также сильное уширение сигналов ионов $\mathrm{Fe}^{+}$при увеличении температуры, наблюдаемое в [18], не позволяет этого сделать, т.е. сделать выбор между указанными ионами пока невозможно. Решить вопрос о природе тригональных центров можно, например, путем исследования изменения интенсивностей их сигналов при варьировании концентрации в образце хрома, железа и никеля. При этом может решиться и вопрос о причине тригонального искажения парамагнитного центра.

В работах $[1,24]$ кристаллы ZnSe с примесью кобальта и железа демонстрировали ЭПР-спектр одиночных ионов $\mathrm{Fe}^{3+}$, т.е. кубических центров с малой величиной начальных расщеплений. В связи с этим максимальная протяженность спектра кубических центров $\mathrm{Fe}^{3+}$ на рис. 2 должна быть меньше ширины сверхтонкой структуры кубических центров $\mathrm{Mn}^{2+}$ [3]. Поэтому все сигналы $\mathrm{Fe}^{3+}$ будут перекрыты сверхтонкой структурой $\mathrm{Mn}^{2+}$. Поскольку из-за спектра $\mathrm{Mn}^{2+}$ сигналов центров $\mathrm{Fe}^{3+}$ не заметно можно лишь утверждать, что в образцах № 1 и 2 интенсивность сигналов кубических центров $\mathrm{Fe}^{3+}$ при комнатной температуре заметно меньше чем для кубических центров $\mathrm{Mn}^{2+}$.

Пиковые интенсивности сигналов первой и второй групп, а также спектра кубических центров $\mathrm{Mn}^{2+}$ в образцах № 1 и 2 при комнатной температуре примерно одинаковы (рис. 2). Спектр ЭПР образца № 3, имеющего заметно меньшие размеры, практически не наблюдается. 


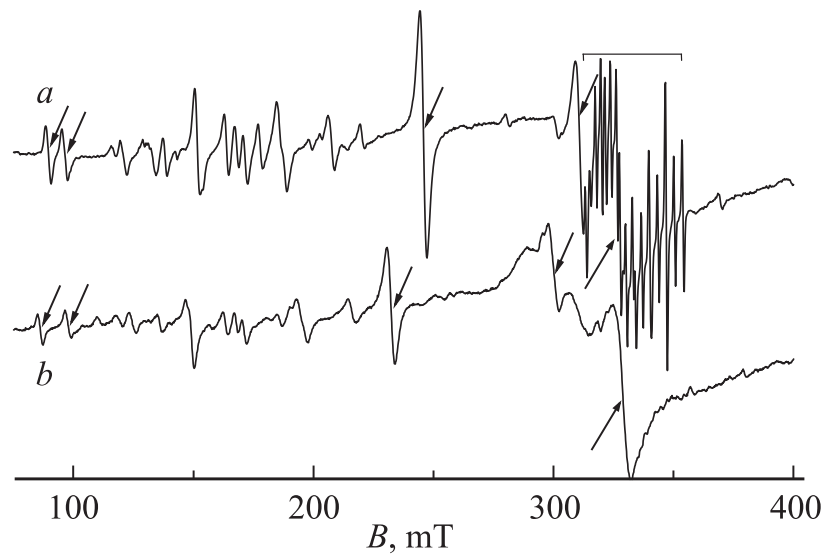

Рис. 9. Вид спектра ЭПР образцов $\mathrm{ZnSe}: \mathrm{Fe}$ вблизи В $\| C_{4}$ при $160 \mathrm{~K}$ на частоте $\sim 9.4 \mathrm{GHz}, a-$ образец № $2, b-$ образец № 3 (ориентации магнитного поля немного отличаются). Нижними стрелками показаны переходы кубического центра $\mathrm{Fe}^{3+}$, верхними - сигналы внутридублетных переходов $( \pm 1 / 2)$ моноклинных центров $\mathrm{Fe}^{3+}$, горизонтальной квадратной скобкой - сверхтонкая структура кубических центров $\mathrm{Mn}^{2+}$.

При понижении температуры (до $\sim 160 \mathrm{~K}$ ) происходит сильный рост интенсивности сигналов центров $\mathrm{Fe}^{3+}$ (как второй группы, так и кубических центров $\mathrm{Fe}^{3+}$ ), обусловленный уменышением их ширины. В связи с этим сигналы тригональных центров (первая группа), ширина которых практически не меняется, выглядят очень слабыми. Возможно, именно указанная особенность не позволила наблюдать междублетные переходы тригональных центров при температуре жидкого гелия (см. разд. 3.2).

Следует заметить, что при $160 \mathrm{~K}$ образец № 2 демонстрирует спектры кубических центров $\mathrm{Fe}^{3+}$ и $\mathrm{Mn}^{2+}$ сравнимой интенсивности, тогда как в спектре образца № 3 сигналы центров $\mathrm{Mn}^{2+}$ практически отсутствуют (рис. 9). Данный факт свидетельствует о неравномерном распределении примеси в кристалле. Как и в работе [1], кроме ЭПР-переходов, обсуждаемых в разд. 3, наблюдаются (особенно при пониженной температуре) слабые не идентифицированные нами сигналы.

\section{5. Заключение}

Техникой ЭПР на разных частотах исследованы монокристаллы селенида цинка, выращенные методом физического транспорта на затравку ZnSe в атмосфере гелия, легированные железом и содержащие значительную концентрацию меди. Наряду с кубическими центрами $\mathrm{Fe}^{3+}$ и $\mathrm{Mn}^{2+}$, а также центрами $\mathrm{Fe}^{2+}$ и $\mathrm{Cr}^{2+}$ обнаружены моноклинные комплексы трехзарядных ионов железа, обусловленные ассоциатами $\mathrm{Fe}^{3+}$ с ионами $\mathrm{Cu}^{+}$, заместившими $\mathrm{Zn}^{2+}$ в ближайшей позиции в направлении $\langle 110\rangle$ на расстоянии $0.4 \mathrm{~nm}$. Наблюдалась квартетная структура сигналов этих центров, обусловленная взаимодействием с ядерными спинами меди. Измерены начальные расщепления указанных центров, определены параметры моноклинного спинового гамильтониана.

Обнаружены и исследованы тригональные центры со спином $3 / 2$. В результате измерения ориентационного поведения положений двух внутридублетных переходов определены параметры спинового гамильтониана. Приведены возможные модели описанных тригональных центров.

\section{Список литературы}

[1] Д.Д. Крамущенко, И.В. Ильин, В.А. Солтамов, П.Г. Баранов, В.П. Калинушкин, М.И. Студеникин, В.П. Данилов, Н.Н. Ильичев, П.В. Шапкин. ФТТ 55, 234 (2013).

[2] G. Colibaba, M. Caraman, I. Evtodiev, S. Evtodiev, E. Goncearenco, D. Nedeoglo, N. Nedeoglo. J. Luminescence 145, 237 (2014).

[3] В.И. Козловский, Ю.В. Коростелин, Я.К. Скасырский. Тезисы докл. XI Рос. конф. по физике полупроводников, Санкт-Петербург (2013). С. 144.

[4] Г.С. Шакуров, Д.С. Пыталев, В.И. Козловский, Ю.В. Коростелин. Матер. XXI Всерос. конф. „Оптика и спектроскопия конденсированных сред“, Краснодар (2015). С. 20.

[5] Y.V. Korostelin, V.I. Kozlovsky. J. Alloys Compounds 371, 25 (2004).

[6] R.S. Title. Phys. Rev. 131, 2503 (1963).

[7] W.C. Holton, M. de Wit, T.L. Estle, B. Dischler, J. Schneider. Phys. Rev. 169, 359 (1968).

[8] N. Sielaff, H. Nelkowski, H. Pradella, J. Sahm, K. Zink. Phys. Status Solidi B 129, K177 (1985).

[9] С.А. Альтшулер, Б.М. Козырев. Электронный парамагнитный резонанс соединений элементов промежуточных групп. Наука, М. (1972). С. 121.

[10] J.T. Vallin, G.D. Watkins. Phys. Rev. B 9, 2051 (1974).

[11] M.E.J. Boonman, W. Mac, A. Twardowski, A. Wittlin, P.J.M. van Bentum, J.C. Maan, M. Demianiuk. Phys. Rev. B 61, 5358 (2000).

[12] А.Т. Лончаков, С.М. Подгорных, В.И. Соколов, Н.Б. Груздев, Г.С. Шакуров. ФТТ 48, 1610 (2006).

[13] Я.Г. Клява. ЭПР-спектроскопия неупорядоченных твердых тел. Зинатне, Рига. (1988). 320 с.

[14] R.W. Kedzie, D.H. Lyons, M. Kestigian. Phys. Rev. 138, A918 (1965).

[15] H.H. Wickman, M.P. Klein, D.A. Shirley. J. Chem. Phys. 42, 2113 (1965).

[16] Н.С. Гарифьянов, М.М. Зарипов. ЖЭТФ 28, 629 (1955).

[17] W.C. Holton, J. Schneider, T.L. Estle. Phys. Rev. 133, A1638 (1964).

[18] S.A. Marshall, S.V. Nistor. Phys. Rev. B 6, 24 (1972).

[19] S.V. Nistor, M. Stefan, D. Schoemaker. Phys. Status Solidi B 214, 229 (1999).

[20] H. Vrielinck, F. Callens, P. Matthys, S.V. Nistor, D. Ghica, D. Schoemaker. Phys. Rev. B 64, 024405 (2001).

[21] М.М. Зарипов, В.С. Кропотов, Л.Д. Ливанова, В.Г. Степанов. ФТТ 9, 209 (1967).

[22] М.М. Зарипов, В.С. Кропотов, Л.Д. Ливанова, В.Г. Степанов. ФТТ 10, 325 (1968).

[23] Л.К. Аминов, Б.З. Малкин. ФТТ 9, 1316 (1967).

[24] J. Dieleman. Philips Res. Rept. 20, 206 (1965). 